\title{
An extended constitutive model for nonlinear reversible ferromagnetic behaviour under magnetomechanical multiaxial loading conditions
}

Cite as: J. Appl. Phys. 121, 053901 (2017); https://doi.org/10.1063/1.4975119

Submitted: 30 September 2016 . Accepted: 18 January 2017 . Published Online: 01 February 2017

Artjom Avakian (iD), and Andreas Ricoeur
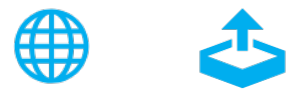

Export Citation

\section{ARTICLES YOU MAY BE INTERESTED IN}

A general one-dimension nonlinear magneto-elastic coupled constitutive model for magnetostrictive materials

AIP Advances 5, 107201 (2015); https://doi.org/10.1063/1.4933024

A general nonlinear magnetomechanical model for ferromagnetic materials under a constant weak magnetic field

Journal of Applied Physics 119, 145103 (2016); https://doi.org/10.1063/1.4945766

A nonlinear constitutive model for Terfenol-D rods

Journal of Applied Physics 97, 053901 (2005); https://doi.org/10.1063/1.1850618

Meet the Next Generation of Quantum Analyzers And Join the Launch Event on November 17th

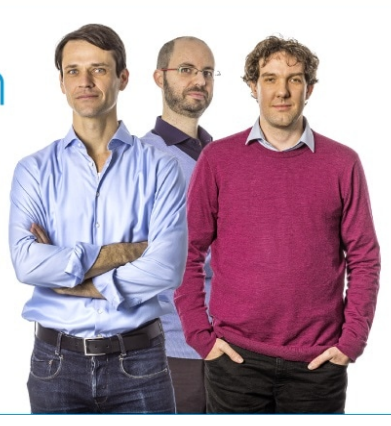




\title{
An extended constitutive model for nonlinear reversible ferromagnetic behaviour under magnetomechanical multiaxial loading conditions
}

\author{
Artjom Avakian ${ }^{\text {a) }}$ and Andreas Ricoeur \\ Institute of Mechanics, University of Kassel, 34125 Kassel, Germany
}

(Received 30 September 2016; accepted 18 January 2017; published online 1 February 2017)

\begin{abstract}
A constitutive modelling of ferromagnetic materials under combined magnetomechanical multiaxial loading with different boundary conditions and a finite element implementation are presented. The phenomenologically motivated model is capable of predicting magnetisation, strain, and stress and is thus suitable, e.g., for applications in multiferroic composites. The approach covers a reversible nonlinear behaviour as it is observed, e.g., in cobalt ferrite and other soft magnetic alloys. Various examples demonstrate the suitability of the model and its numerical implementation and give an insight into the behaviour of soft magnets, exposed to different boundary conditions or being embedded into other compliant materials. Published by AIP Publishing.
\end{abstract}

[http://dx.doi.org/10.1063/1.4975119]

\section{INTRODUCTION}

\section{A. Reversible constitutive behaviour and modelling goals}

The principles of ferromagnetism are well understood nowadays (Bergmann and Schaefer, 2005; Bozorth, 1951; Du Trémolet de Lacheisserie et al., 2005; Kittel, 2006; Morrish, 2001; Stefanita, 2012), although there are still research activities in the physics community. For engineering applications, however, the knowledge of the macroscopic material behaviour is more essential than a deep understanding of the physics on the atomic scale. Magnetostriction is technically exploited in actuation systems, and there is a variety of applications for permanent magnetic fields of poled ferromagnetic devices. New concepts combine ferromagnetic and ferroelectric phases in the so-called multiferroic composites (Bibes and Barthélémy, 2008; Buchanan, 2004; Eerenstein et al., 2007; Fiebig, 2005; Hill, 2000; Lu et al., 2011; Nan, 1994; Nan et al., 2008; Scott, 2007) in order to induce a coupling of electric and magnetic fields. All these applications require the knowledge of the constitutive behaviour of the employed ferromagnetic material.

Plots of the magnetic induction or magnetisation vs. the magnetic field are mostly provided by manufacturing companies. The so called hard ferromagnetic materials exhibit a pronounced hysteresis behaviour as it is known e.g., from ferroelectric materials. Soft ferromagnets, on the other hand, show an almost reversible nonlinear characteristic as it is known e.g., from electrostriction. Even two identical chemical compositions can, in this context, exhibit qualitatively different features (Bhame and Joy, 2007, 2008; Etier et al., 2012; Feltin and Pileni, 1997; Lu et al., 2007), depending, for e.g., on the sintering conditions. In Fig. 1, a typical plot of magnetisation $\vec{M}$ vs. magnetic field $\vec{H}$ is depicted showing four regions, each one on the microscale being attributed to different stages of domain activity or Bloch wall motion, respectively (Bergmann and Schaefer, 2005; Bozorth, 1951;

a)artjom.avakian@uni-kassel.de
Du Trémolet de Lacheisserie et al., 2005; Kittel, 2006; Morrish, 2001; Stefanita, 2012). In Fig. 2, a specific magnetisation $\vec{m}$ and strain $\lambda$ are plotted vs. the magnetic field for a cobalt ferrite sample. In fact, the material shows nearly reversible behaviour, as expected of soft magnetic alloys.

In this paper, an approach is presented for the constitutive modelling of soft ferromagnetic materials, starting from a thermodynamical potential, providing a reversible nonlinear behaviour. Thermodynamical consistency is important for phenomenological models, in order to guarantee their general applicability beyond the few experimental conditions, which are required to identify the model parameters. The ferromagnetic model presented here has been developed focussing on three issues: first of all, realistic loading scenarios shall be covered, i.e., a specimen is allowed to be exposed to mechanical and magnetic fields simultaneously, involving non-homogeneous Dirichlet or Neumann boundary conditions as well as more complex interface conditions due to adjacent compliant media. Second, multiaxial states of stress and strain have to be taken into account for the sake of applications to real smart devices, implying a tensorial representation, suitable for a finite element (FE) implementation. Eventually, the identification of the model parameters should be straightforward, uniquely emanating from easily accessible experimental data.

Based on the weak formulation of balance laws, the constitutive model has been implemented into a FE code to solve the complex boundary value problems, and to provide a numerical tool being able to predict the multifieldbehaviour of smart devices and to improve their performance. The focus here being on the constitutive behaviour, simple bulk specimens under uni- or multiaxial magnetomechanical loading are chosen to demonstrate and discuss the basic effects observed at ferromagnetic materials.

\section{B. State of the art}

Phenomenological constitutive models, adapting multiple empirical parameters to experimental data, have been 


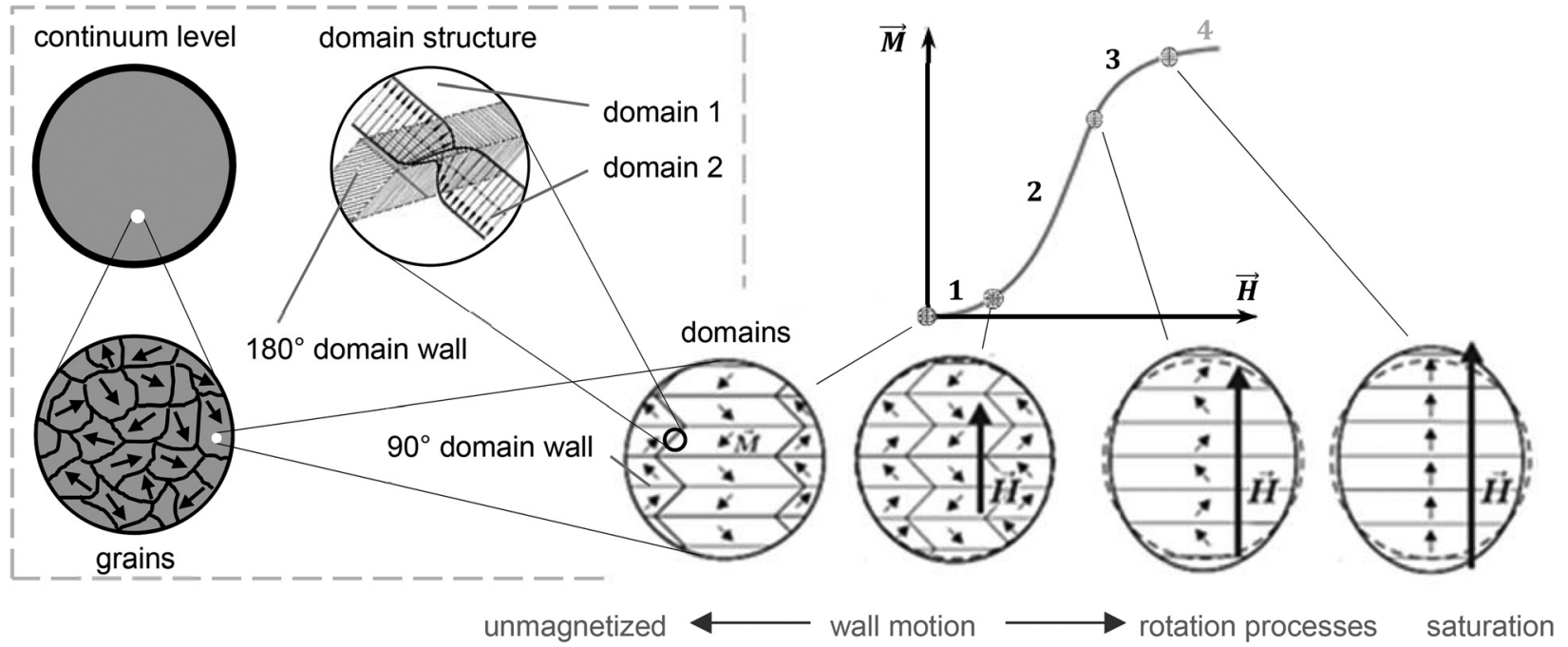

FIG. 1. Different scales of describing ferromagnets (left) and magnetisation curve separated into four regions with associated domain patterns.

developed with and without internal variables, in order to describe the irreversible (Carman and Mitrovic, 1995; Kiefer and Lagoudas, 2004; Linnemann et al., 2009; Miehe et al., 2011a; 2011b; Xu et al., 2013; Wang et al., 2016; Shi et al., 2016) or reversible ferromagnetic and magnetostrictive behaviour. Concerning models for the nonlinear reversible behaviour, the simplest extension of linearity is given by the standard square model (Carman and Mitrovic, 1995; Wan et al., 2003; Wan and Zhong, 2004). It is capable of describing the symmetry of strain with respect to a change of sign in the magnetic field and the zero gradient for vanishing fields, it however fails to reproduce the saturation for larger magnetic loads. Concerning models with saturation, in (Wan et al., 2003) two approaches are suggested, the so-called hyperbolic tangent model (HT) and the density of domain switching model (DDS). Whereas the HT overestimates the magnetostriction by $40 \%$, the DDS leads to its underestimation by up to $30 \%$, depending on the mechanical pre-load. In (Zheng and Liu, 2005) a model is presented, which is in good agreement with experimental findings, however different ranges of the curves are described by separate equations. None of these papers deals with a general multiaxial tensorial representation of the constitutive equations or a FE implementation within an electro-magneto-mechanical framework. Moreover, most of the material laws have specifically been developed and verified for the behaviour of Terfenol-D.

Lately, Avakian and Ricoeur published a phenomenological multi-axial tensorial constitutive model and showed results of FE simulations of different magnetomechanical boundary value problems (Avakian and Ricoeur, 2016). The major drawback of this approach is that magnetic and mechanical quantities, being well predicted for mechanical Neumann boundary conditions, are inaccurately reproduced for Dirichlet conditions or equilibrium conditions at material interfaces. Particularly the latter aspect is crucial, however, if applications of ferromagnetic modelling in multiferroic composites are addressed. The improved approach, presented in this paper, is capable of accurately predicting the constitutive behaviour of soft ferromagnets under arbitrary mixed multiaxial boundary conditions. Being formulated within a continuum mechanical framework, the model is suitable for implementation into a FE code, which was done using the UEL (User element)-interface in ABAQUS. Numerical examples demonstrate the suitability and efficiency of the approach. More references related to ferromagnetic constitutive modelling have been compiled in Avakian and Ricoeur (2016).
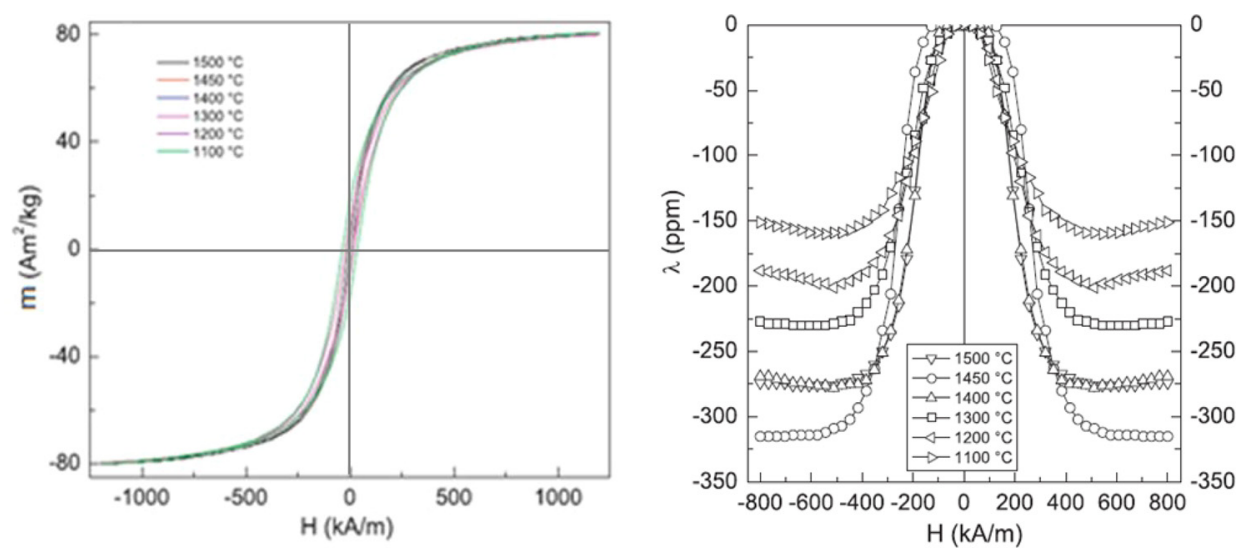

FIG. 2. Specific magnetisation and strain vs. magnetic field for cobalt ferrite at room temperature with different sintering temperatures (adapted from Mohaideen and Joy, 2014). 


\section{CONSTITUTIVE FERROMAGNETIC MODEL AND BALANCE EQUATIONS}

For the electro- and magnetostatic case $\left(\dot{B}_{k}, \dot{D}_{l}=0\right.$, where $B_{k}$ and $D_{l}$ are the magnetic induction and electric displacement), the scalar electric and magnetic potentials $\varphi^{e l}$ and $\varphi^{m}$ are motivated from the Maxwell equations (Jackson, 1998). Their gradients yield electric $E_{l}$ and magnetic $H_{k}$ fields (Vanderlinde, 2005), just as displacements $u_{i}$ and strain $\varepsilon_{i j}$ are related for infinitely small deformations

$$
\varepsilon_{i j}=\frac{1}{2}\left(u_{i, j}+u_{j, i}\right), \quad E_{l}=-\varphi_{, l}^{e l}, \quad H_{k}=-\varphi_{, k}^{m} .
$$

Dots on variables denote the time derivatives. Here, as in the following, the analytical notation is used introducing lower case indices taking values $1,2,3$ and implying summation over repeated indices. In this paper, electrical quantities and properties of the ferromagnetic material are considered likewise, due to the intended application of the model to multiferroic composites. To define boundary value problems, the balance equation of momentum

$$
\sigma_{i j, j}+b_{i}=\rho \ddot{u}_{i},
$$

has to be considered besides the remaining two Maxwell equations, not being trivially satisfied within a quasistatic framework. In the Eq. (2) $\sigma_{i j}$ is the stress tensor. Inertia effects and specific body forces will be neglected in the intended applications, i.e., $b_{i}=0, p \ddot{u}_{i}=0$ in Eq. (2). Since the free electric volume charges are assumed not to be present in a dielectric material, the mechanical and magnetoelectrostatic balance equations can be specified as

$$
\sigma_{i j, j}=0, \quad D_{l, l}=0 \quad B_{k, k}=0 .
$$

For the FE implementation, the weak formulations of these differential equations are required, which can be looked-up, for e.g., in (Avakian and Ricoeur, 2016) or (Avakian et al., 2015). Cauchy's theorem, introducing tractions $t_{i}$, is generalized providing the relations

$$
t_{i}=\sigma_{i j} n_{j}, \quad \omega_{s}^{e l}=-D_{k} n_{k}, \quad \omega_{s}^{m}=-B_{l} n_{l},
$$

where $\omega_{s}^{e l}$ is the surface charge density, and $\omega_{s}^{m}$ is the part of the magnetic flux along the surface normal $n_{l}$ of the Neumann type boundary $S_{\omega}$.

The constitutive behaviour of the ferromagneticdielectric material is assumed to be governed by the thermodynamic potential $\bar{\Psi}$

$$
\begin{aligned}
\bar{\Psi}\left(\sigma_{p}, E_{l}, H_{k}\right)= & -\frac{1}{2} s_{11} \sigma_{1} \sigma_{1}-s_{12} \sigma_{1} \sigma_{2}-\frac{1}{2} s_{22} \sigma_{2} \sigma_{2}-s_{66} \sigma_{6} \sigma_{6}-\frac{1}{2} \kappa_{11} E_{1} E_{1}-\frac{1}{2} \kappa_{22} E_{2} E_{2} \\
& -\frac{1}{2} \mu_{11}^{0} H_{1} H_{1}-\frac{\eta_{1}}{1+\zeta_{1} H_{1}^{-3}} \sigma_{1}-\frac{\eta_{2}}{1+\zeta_{2} H_{1}^{-3}} \sigma_{2}-\rho\left\{H_{1}-\xi \ln \left(\xi+H_{1}\right)\right\},
\end{aligned}
$$

where stress, electric, and magnetic fields are chosen as independent variables. Here, $s_{p q}, \kappa_{l n}$, and $\mu_{11}^{0}$ are the coefficients of compliance, dielectric and magnetic permeability tensors, respectively. It is feasible to develop the material model based on stress and magnetic field, since these are the quantities which are commonly controlled in experiments, where, for e.g., stresses are zero due to free boundaries. In this section, the Voigt notation is applied to higher order tensors, so, for e.g., $\sigma_{6}$ is the shear stress $\sigma_{12}$. Essential features of magnetisation and magnetostriction are appropriately described adapting the constant coefficients $\eta_{i}, \zeta_{i}, \rho$, and $\xi$ to experimental curves. Table III in the Appendix indicates how to identify the material parameters from experimental data.

Equation (5) has been formulated in a local coordinate system where the $x_{1}$-axis is attached to the vector of the $H$ field. Thus, $\mathrm{H}_{2}$ does not appear in the potential. The easy axis locally always points in the direction of the magnetic field, since the reversibility, in connection with a vanishing remanence, leads to an immediate magnetisation even at low field intensities. Consequently, the $x_{1}$-axes of the local coordinate systems are always attached to the direction of magnetisation and magnetic induction, and the material tensors are sparsely populated in these coordinate systems. The potential according to Eq. (5), for curved magnetic flux lines being valid locally and adjusted to the local coordinates, thus contains only these coefficients. In contrast to hard magnetic materials, stresses do not control the direction of magnetisation and the easy axis, respectively, in fact having an impact only on the local magnitudes of the $\mathrm{H}$-field. If boundary conditions are fundamentally changed during the loading process, generally an iteration is required in each load step, adapting the evolving magnetic flux lines and the local coordinate systems to one another. Finally, all fields are transformed into global coordinates, where the material tensors in general are fully populated.

The superscript in the magnetic permeability $\mu_{11}^{0}$ indicates a constant magnitude in contrast to the function $\bar{\mu}_{11}\left(\sigma_{p}, H_{k}\right)$, see Fig. 3. The denominators in Eq. (5) cannot be zero since $\zeta_{1}$ and $\zeta_{2}$ are always positive, requiring a negative value of $H_{1}$ for a division by zero. This is not possible,

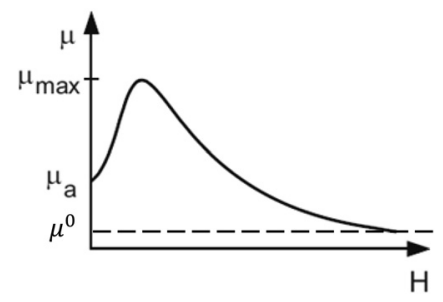

FIG. 3. Typical behaviour of permeability vs. H-field for a ferromagnetic material (Kallenbach et al., 2012, $\mu^{0}$ has been added, $\mu_{\mathrm{a}}$ is the initial permeability) 
however, since the local coordinate system is always adapted to the $\mathrm{H}$-field such that $H_{1}>0$. The general constitutive behaviour is obtained by differentiation of Eq. (5) according to

$$
\begin{aligned}
\dot{\varepsilon}_{p}\left(\dot{\sigma}_{q}, \dot{E}_{l}, \dot{H}_{k}\right) & =\frac{-\partial^{2} \bar{\Psi}}{\partial \sigma_{p} \partial \sigma_{q}} \dot{\sigma}_{q}+\frac{-\partial^{2} \bar{\Psi}}{\partial \sigma_{p} \partial E_{l}} \dot{E}_{l}+\frac{-\partial^{2} \bar{\Psi}}{\partial \sigma_{p} \partial H_{k}} \dot{H}_{k}, \\
\dot{D}_{l}\left(\dot{\sigma}_{p}, \dot{E}_{n}, \dot{H}_{k}\right) & =\frac{-\partial^{2} \bar{\Psi}}{\partial E_{l} \partial \sigma_{p}} \dot{\sigma}_{p}+\frac{-\partial^{2} \bar{\Psi}}{\partial E_{l} \partial E_{n}} \dot{E}_{n}+\frac{-\partial^{2} \bar{\Psi}}{\partial E_{l} \partial H_{k}} \dot{H}_{k}, \\
\dot{B}_{k}\left(\dot{\sigma}_{p}, \dot{E}_{l}, \dot{H}_{m}\right) & =\frac{-\partial^{2} \bar{\Psi}}{\partial H_{k} \partial \sigma_{p}} \dot{\sigma}_{p}+\frac{-\partial^{2} \bar{\Psi}}{\partial H_{k} \partial E_{l}} \dot{E}_{l}+\frac{-\partial^{2} \bar{\Psi}}{\partial H_{k} \partial H_{m}} \dot{H}_{m},
\end{aligned}
$$

where the material coefficients, for e.g., the compliances $s_{11}$, $s_{12}$, are assumed to be constant within the incremental changes of state, and thus the rate dependent constitutive framework is given by

$$
\begin{aligned}
\dot{\varepsilon}_{p}\left(\dot{\sigma}_{q}, \dot{H}_{k}\right) & =s_{p q} \dot{\sigma}_{q}+\bar{q}_{k p}\left(H_{k}\right) \dot{H}_{k} \\
\dot{D}_{l}\left(\dot{E}_{n}\right) & =\kappa_{l n} \dot{E}_{n}, \\
\dot{B}_{k}\left(\dot{\sigma}_{p}, \dot{H}_{m}\right) & =\bar{q}_{k p}\left(H_{m}\right) \dot{\sigma}_{p}+\bar{\mu}_{k m}\left(\sigma_{p}, H_{m}\right) \dot{H}_{m} .
\end{aligned}
$$

Here, $\bar{q}_{k p}$ represents the magnetostrictive tensor. Eq. (7) represents the nonlinear reversible changes of state since the material tensors are single-valued functions of the independent variables. The electric displacement only depends on the electric field, in a ferromagnetic material not being strongly coupled with mechanical or magnetic fields. Having a closer look, the dielectric properties are controlled by magnetic fields to a certain extent, the latter rotating the axis of transversal isotropy. This effect is intrinsically taken into account by the proposed model due to the local coordinate systems, being attached to the magnetic flux lines, as outlined above. In Eq. (7), a bar is added to the magnetic permeability $\bar{\mu}_{k m}\left(\sigma_{p}, H_{m}\right)$ as before and magnetostrictive coefficients $\bar{q}_{k p}\left(H_{k}\right)$ to distinguish from quantities based on a different potential. Due to the tensorial representation, Eq. (7) allows for multiaxial loading. The responses, for e.g., in the $x_{1}$-direction are obtained as

$$
\begin{gathered}
\dot{\varepsilon}_{1}\left(\dot{\sigma}_{q}, \dot{H}_{k}\right)=s_{11} \dot{\sigma}_{1}+s_{12} \dot{\sigma}_{2}+3 \frac{\eta_{1} \zeta_{1} H_{1}^{2}}{\left(\zeta_{1}+H_{1}^{3}\right)^{2}} \dot{H}_{1}, \\
\dot{D}_{1}\left(\dot{E}_{n}\right)=\kappa_{11} \dot{E}_{1},
\end{gathered}
$$

In general, all material coefficients depend on the three independent variables. Experimental observations, however, put this thermodynamical requirement into perspective, showing, for e.g., a noticeable nonlinearity of the stress-strain curve only for giant magnetostrictive materials. In the potential of Eq. (5) and the constitutive relations of Eq. (7), the magnetostrictive constants are functions of just the magnetic field, and the magnetic permeability is a function of both magnetic field and stress

$$
\begin{gathered}
\bar{q}_{11}=3 \frac{\eta_{1} \zeta_{1} H_{1}^{2}}{\left(\zeta_{1}+H_{1}^{3}\right)^{2}}, \quad \bar{q}_{12}=3 \frac{\eta_{2} \zeta_{2} H_{1}^{2}}{\left(\zeta_{2}+H_{1}^{3}\right)^{2}} . \\
\bar{\mu}_{11}=\mu_{11}^{0}+\frac{6 \eta_{1} \zeta_{1} H_{1}\left(\zeta_{1}-2 H_{1}^{3}\right) \sigma_{1}}{\left(\zeta_{1}+H_{1}^{3}\right)^{3}}+\frac{6 \eta_{2} \zeta_{2} H_{1}\left(\zeta_{2}-2 H_{1}^{3}\right) \sigma_{2}}{\left(\zeta_{2}+H_{1}^{3}\right)^{3}} \\
+\frac{\rho \xi}{\left(\xi+H_{1}\right)^{2}} .
\end{gathered}
$$

The constant part of the magnetic permeability $\mu_{11}^{0}$ is intended to represent the linear behaviour of the B-field, once the spontaneous magnetisation is saturated at large magnetic fields, following the general relation:

$$
\begin{aligned}
B_{k} & =\mu_{0}\left(\delta_{k m}+\chi_{k m}\right) H_{m}+M_{k}^{s p}=\mu_{0} \mu_{k m}^{r} H_{m}+M_{k}^{s p} \\
& =\mu_{k m} H_{m}+M_{k}^{s p} .
\end{aligned}
$$

Here, $\delta_{k m}$ is the Kronecker identity tensor, $\chi_{k m}$ the magnetic susceptibility, $\mu_{0}$ the magnetic permeability of vacuum, and $\mu_{k m}^{r}$ are the coefficients of relative permeability of the material $(\geq 1)$. From literature it is known that the coefficients $\mu_{k m}$ depend on the applied $\mathrm{H}$-field and that a convergence towards a constant value $\mu_{k m}^{0}$ is observed for larger magnetic loads, i.e., in the saturation regime, see Fig. 3. The variable part of the permeability and the spontaneous magnetisation $M_{k}^{s p}$ in Eq. (13) are thus represented by the last three terms in Eq. (12), whereas $\mu_{11}^{0}$ is the saturation value depicted in the figure. It solely remains when the other terms vanish for sufficiently large magnetic loads. Its magnitude is much smaller than those permeabilities typically found in charts, the latter representing values for much lower magnetic fields, where $\mu_{\mathrm{a}}<\mu<\mu_{\max }$. A parameter $\varpi=\mu / \mu^{0}$ is thus introduced to provide a suitable $\mu_{11}^{0}$, based on values of relative permeabilities $\mu_{11}^{r}$ available from literature:

$$
\mu_{11}^{0}=\frac{\mu_{11}}{\varpi}=\frac{1}{\varpi} \mu_{11}^{r} \mu_{0}
$$


The parameter $\varpi$ is calibrated on the basis of experimental plots, typically taking values in the range of a few tens, see Appendix.

The constitutive model based on constant coefficients $\zeta_{1}, \zeta_{2}$, and $\xi$ is suitable for Neumann type boundary conditions. The evolution of stress, which is inevitably associated with, for e.g., a Dirichlet condition, is however not appropriately taken into account with constant coefficients. A more sophisticated model thus replaces the constant coefficients $\zeta_{1}, \zeta_{2}$, and $\xi$ by variables depending on stresses and their evolution

$$
\begin{aligned}
\zeta_{1} & =\zeta_{1}^{0}+\zeta_{1}^{\sigma}\left(\sigma_{1}-\sigma_{2}\right), \zeta_{2}=\zeta_{2}^{0}+\zeta_{2}^{\sigma}\left(\sigma_{1}-\sigma_{2}\right), \\
\xi & =\xi^{0}+\xi^{\sigma}\left(\sigma_{1}-\sigma_{2}\right)+\hat{\xi}^{\sigma}\left(d \sigma_{1}-d \sigma_{2}\right),
\end{aligned}
$$

where $d \sigma_{i}$ denotes the change of the normal stress $\sigma_{i}$ in each load increment and $\zeta_{2}^{\sigma}=\zeta_{1}^{\sigma}\left(\left|\sigma_{1}^{s}\right| /\left|\sigma_{2}^{s}\right|\right)$. Here, $\left|\sigma_{1}^{s}\right|$ and $\left|\sigma_{2}^{s}\right|$ represent the absolute values of saturated normal stresses, which can be attained applying realistic magnetic loads. Unfortunately, their magnitudes are not available from literature. Therefore, both values have been calculated for plane stress conditions, based on the saturation strains $\varepsilon_{1}^{s} \approx \eta_{1}$ and $\varepsilon_{2}^{s} \approx \eta_{2}$ in connection with the stiffness $c_{p q}$, see Appendix Eq. (A1). The last term of $\xi$ in Eq. (15) involving the parameter $\hat{\xi}^{\sigma}$ is crucial, if Dirichlet boundary conditions or adjacent compliant phases shall be considered. Thus, it is inevitable for the investigation of multiferroic composites, where a ferromagnetic phase may be embedded into a ferroelectric one. For constant mechanical loads $\sigma_{1}, \sigma_{2}$ the term vanishes. For displacement boundary conditions, both $\sigma_{1}, \sigma_{2}$ and $d \sigma_{1}, d \sigma_{2}$ emanate from the solution of the boundary value problem.

Introducing the parameters $\zeta_{1}\left(\sigma_{1}, \sigma_{2}\right), \zeta_{2}\left(\sigma_{1}, \sigma_{2}\right)$, and $\xi\left(\sigma_{1}, \sigma_{2}, d \sigma_{1}, d \sigma_{2}\right)$, the Maxwell relations of thermodynamics are still satisfied, i.e.,

$$
\bar{q}_{k p}=-\frac{\partial^{2} \bar{\Psi}}{\partial \sigma_{p} \partial H_{k}}=\frac{\partial B_{k}}{\partial \sigma_{p}}=\frac{\partial \varepsilon_{p}}{\partial H_{k}} .
$$

Results of the constitutive model will be investigated in Sec. III. For the sake of an efficient numerical implementation, the independent mechanical variable is changed from stress to strain, in principle taking another thermodynamical potential $\tilde{\Psi}\left(\varepsilon_{p}, E_{l}, H_{k}\right)$ as a basis. Accordingly, the material tensors are subject to the following transformations:

$$
c_{p q}=s_{p q}^{-1}, \tilde{q}_{k p}=\bar{q}_{k q} c_{q p}, \tilde{\mu}_{k m}=\bar{\mu}_{k m}-\bar{q}_{k q} c_{q p} \bar{q}_{m p} .
$$

The constitutive equations for the modified set of independent variables are thus given as

$$
\begin{aligned}
\dot{\sigma}_{p}\left(\dot{\varepsilon}_{q}, \dot{H}_{k}\right) & =c_{p q} \dot{\varepsilon}_{q}-\tilde{q}_{k p}\left(\varepsilon_{q}, H_{k}\right) \dot{H}_{k}, \\
\dot{D}_{l}\left(\dot{E}_{n}\right) & =\kappa_{l n} \dot{E}_{n}, \\
\dot{B}_{k}\left(\dot{\varepsilon}_{p}, \dot{H}_{m}\right) & =\tilde{q}_{k p}\left(\varepsilon_{p}, H_{m}\right) \dot{\varepsilon}_{p}+\tilde{\mu}_{k m}\left(\varepsilon_{p}, H_{m}\right) \dot{H}_{m} .
\end{aligned}
$$

Discarding $\mu_{11}^{0}$ in Eqs. (12), (17), and (18) yield the magnetic polarization $M_{k}^{s p}$ according to Eq. (13) instead of $B_{k}$. The specific magnetisation $m_{k}$, which is commonly depicted in experimental plots, is finally obtained as

$$
m_{k}=\mu_{0}^{-1} \varrho^{-1} M_{k}^{s p},
$$

where $\varrho$ denotes the mass density of the material.

\section{RESULTS}

The constitutive model according to Sec. II has been implemented within the framework of the FE method, see, e.g., Avakian and Ricoeur (2016) or Avakian et al. (2015). Due to the incremental formulation according to Eq. (18), the range of the magnetic load $H \in\left[0, H^{\max }\right]$ is divided into load increments $\Delta H$, controlling the evolution of the magnetomechanical fields in each load step, e.g., the stress evolution $d \sigma_{i}$ decreasing or increasing according to the choice of the numerical parameter $\Delta H$. Accordingly, $\hat{\xi}^{\sigma}$ in Eq. (15) has to be adapted to the load increment $\Delta H$ according to

$$
\hat{\xi}^{\sigma}=\hat{\xi}_{0}^{\sigma} \frac{H^{\max }}{\Delta H},
$$

where $\hat{\xi}_{0}^{\sigma}$ depends on the material and is determined from fitting numerical to experimental magnetization plots under clamped conditions, see Table III. Cobalt ferrite $\left(\mathrm{CoFe}_{2} \mathrm{O}_{4}\right)$ is employed as an example of nonlinear reversible soft magnetic behaviour. The material parameters used for the calculations are outlined in the Appendix and $\Delta H=100 \mathrm{~A} / \mathrm{m}$ was chosen. Furthermore, a generalized state of plane stress is assumed.

In Figure 4, results are presented for stress-free boundaries, implicating constant values $\zeta_{1}=\zeta_{1}^{0}, \zeta_{2}=\zeta_{2}^{0}$, and $\xi=\xi^{0}$. As expected, the curves are nonlinear but reversible. They agree well with those in experiments, without
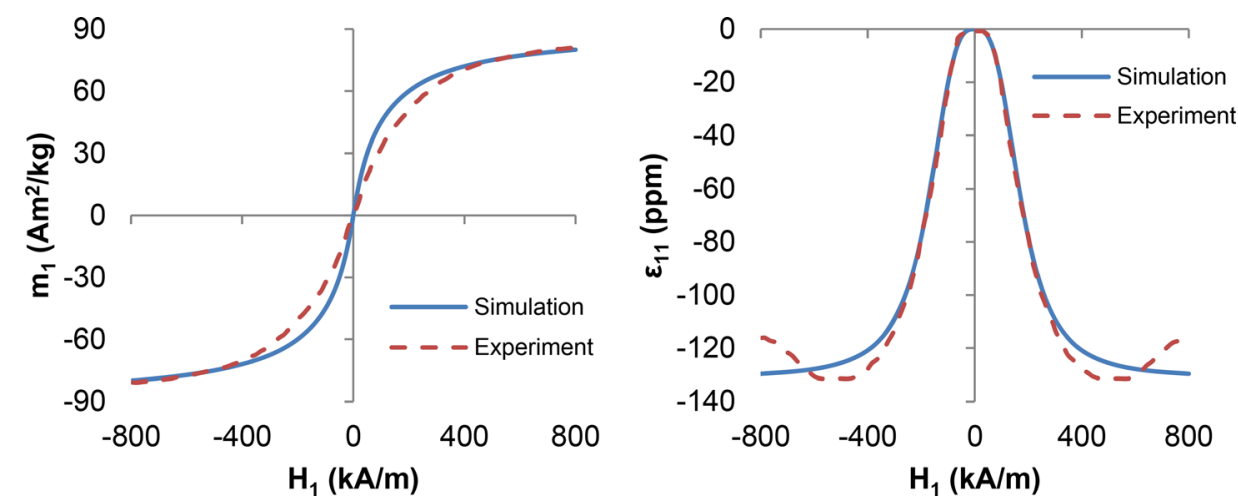

FIG. 4. Experimental data (Bhame and Joy, 2006) and numerical results, left: specific magnetisation, right: strain vs. magnetic field. 

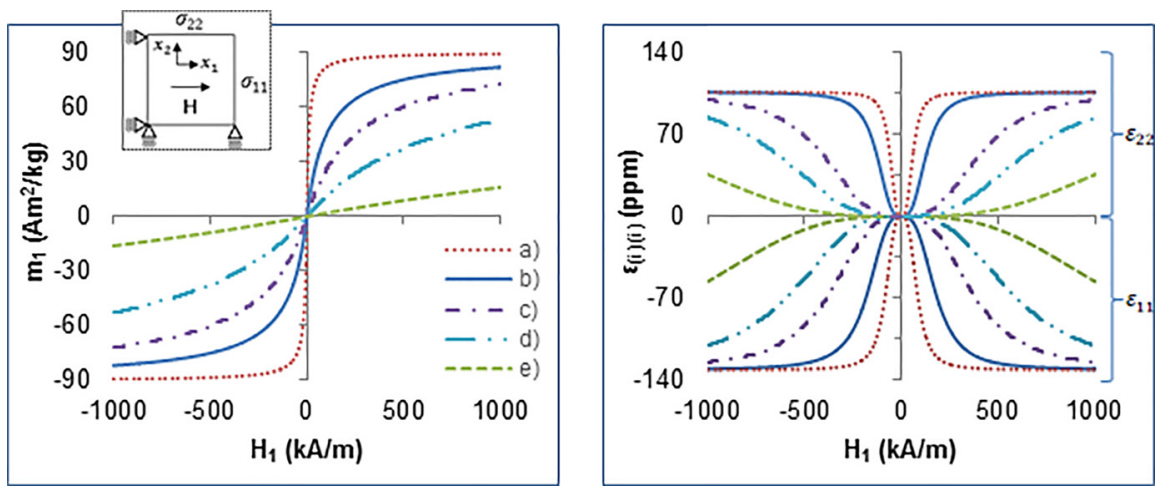

FIG. 5. Numerical results at combined magnetomechanical loading, left: specific magnetisation, right: strain vs. magnetic field at constant stress a) $\sigma_{11}=0$ and $\left.\sigma_{22}=15, \mathrm{~b}\right) \sigma_{11}=0$ and $\sigma_{22}=0$, c) $\sigma_{11}=0$ and $\sigma_{22}=-0.5$, d) $\sigma_{11}=-13$ and $\sigma_{22}=-15$, e) $\sigma_{11}=0$ and $\sigma_{22}=-15 \mathrm{MPa}$.

reproducing every detail of the complex behaviour. The specific magnetisation $m_{k}$ according to Eq. (19) in the left figure is determined with the density $\varrho=5.3 \mathrm{~g} / \mathrm{cm}^{3}$.

In Figure 5, the effect of a superimposed mechanical load on the ferromagnetic and magnetostrictive properties in terms of the magnetisation/strain-magnetic field curves is investigated. In contrast to Fig. 4, the more sophisticated approach has been applied, where $\zeta_{1}, \zeta_{2}$, and $\xi$ depend on the stresses according to Eq. (15). The solid blue lines (b) represent a pure magnetic loading in $x_{1}$-direction, whereas the other lines stand for the combined magnetomechanical loading. The lines with the negative values $\varepsilon_{(i)(i)}$ (the braces are introduced to repeal the summation over repeated indices) represent the strain $\varepsilon_{11}$ along the axis of the magnetic field, whereas those with positive values represent the perpendicular strain $\varepsilon_{22}$. The plots are in agreement to what is expected intuitively. The tensile stress in $x_{2}$-direction supports the magnetic field and leads to a saturation at lower magnetic loads, whereas a compressive stress in that direction acts contrariwise. A compressive stress in the direction of the magnetic field, on the other hand, supports the magnetic loading. Furthermore, the absolute values of the strain are larger in the direction of the magnetic field than perpendicular to it, i.e., $\left|\varepsilon_{11}\right|>\varepsilon_{22}$.

Figure 7 (left) shows the volume averaged specific magnetisation $\left\langle m_{1}\right\rangle$ of the ferromagnetic material for magnetic loading by $\mathrm{H}$-fields and different mechanical boundary conditions depicted in Fig. 6. The solid blue lines a) in Fig. 7 (left) and b) in Fig. 5 (left) are identical, representing the unclamped stress-free state. The dashed line g), on the other hand, represents the other limiting case of clamped boundaries according to the sketch i) in Fig. 6. Compared to the case of free boundaries (a), as expected, the magnitude of the magnetisation is significantly lower. Physically, this feature is due to the compressive stress induced by the clamping, reducing the mobility of domain walls, as depicted in Section I.

In order to simulate the constraints in between these limiting cases, two compound structures are investigated, consisting of two dissimilar materials, see sketches ii) and iii) in Fig. 6. These specific cases of embedded magnetostrictives are crucial for applications of the constitutive model to composite structures and could not be appropriately reproduced by the basic model in (Avakian and Ricoeur, 2016). The same holds for the clamped case, also requiring the model extension. The compound structure consists of a ferromagnetic part and one or two elastic, non-functional bodies denoted as phases 2 and 3. In contrast to the fixed bearings in (i), the whole system is simply supported by loose bearings allowing for displacements along the slits in the symbols, while perpendicular displacements are suppressed. The interfaces are assumed to be perfect, satisfying the usual continuity conditions. The functional phase itself is exposed to a magnetic field. In (ii), where just one edge is connected to a compliant second phase, the mechanical boundary condition and magnetic loading induce an uniaxial stress state in the ferrite, since the upper horizontal edge gives way in the $x_{2}$ direction. In (iii), where the active material is embedded

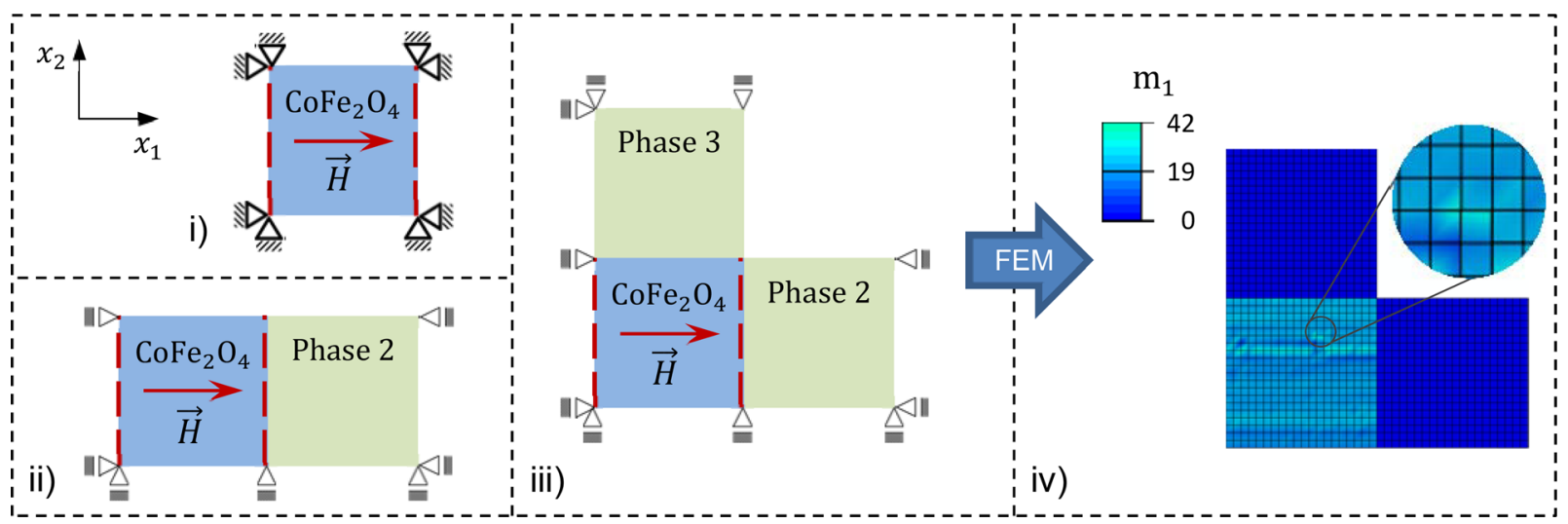

FIG. 6. (i)-(iii) Magnetomechanical boundary conditions, magnetic potential gradients are imposed in between the red dashed lines delimiting the $\mathrm{CoFe}_{2} \mathrm{O}_{4}$ phase, phases 2 and 3 are non-magnetostrictive elastic; (iv): magnetisation $\left[\mathrm{Am}^{2} / \mathrm{kg}\right]$ from FE-calculation with boundary conditions (iii) at $H_{1}=1 \mathrm{MA} / \mathrm{m}$. 

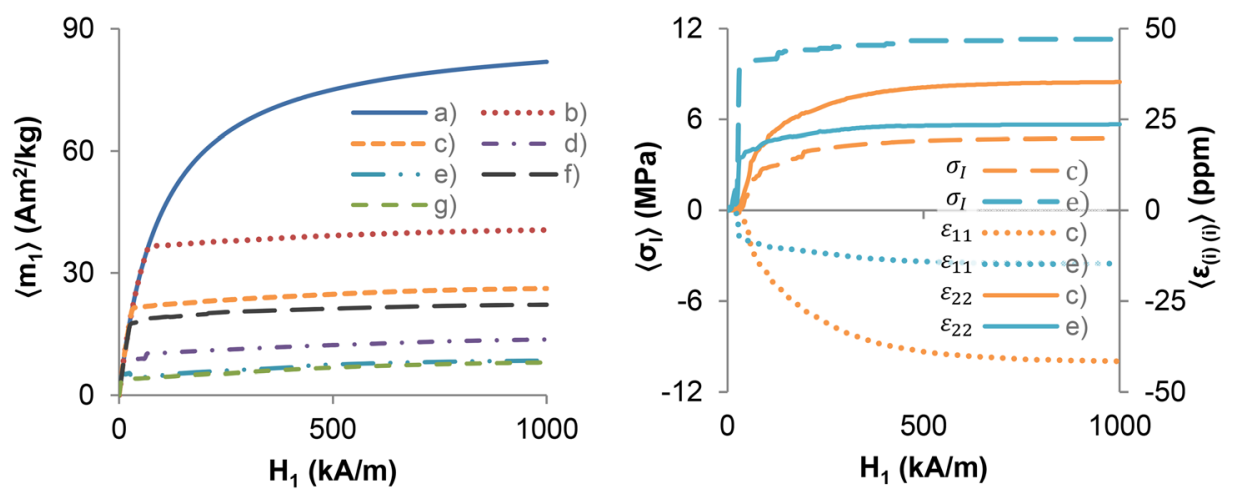

FIG. 7. Volume averaged specific magnetisation (left), average maximum principal stress and strain (right) at different boundary conditions: (a) stress-free $\quad \sigma_{11}=0 \quad$ and $\quad \sigma_{22}=0$, (b)-(e) laminated structure with different Young's moduli of phase 2, see Fig. 6(ii), i.e., (b) 1.12, (c) 11.2, (d) 1120, (e) $112000 \mathrm{MPa}$, (f) compound structure, see Fig. 6(iii), with Young's modulus $11.2 \mathrm{MPa}$ of phases 2 and 3 , and $(\mathrm{g})$ rigid clamping, see Fig. 6(i).

from two sides, a biaxial stress state is evoked, due to the compliant phases 2 and 3 being akin to the conditions in multiferroic composites.

The plots (b) to (e) in Fig. 7 are associated with case (ii), whereas the plot (f) stems from case (iii). The figure shows the behaviour of the $\mathrm{CoFe}_{2} \mathrm{O}_{4}$ for different stiffnesses of the non-functional phases. Volume averages of the maximum principal stress $\left\langle\sigma_{I}\right\rangle$ and the strains $\left\langle\varepsilon_{(i)(i)}\right\rangle$ are plotted for the two variants (c) and (e). Considering at first the unilateral embedding it is obvious that its elastic modulus has a nonlinear impact on the magnetisation. As expected, the rigidly clamped case (f) is asymptotically approached for increasing stiffnesses, once a threshold value of induced stress is attained. This threshold is associated with a critical intensity of the H-field which, of course, decreases with increasing stiffness of phase 2 .

Due to its contraction along the $x_{1}$-axis, tensile stress is predominant in the magnetostrictive, leading to positive maximum principal stress. For the stiffer phase 2, stresses are much larger than for the softer variant. Both stress and magnetisation are observed to approach the plateau values at comparatively low magnetic loads, in particular, for the case with the larger elastic modulus of phase 2 . The average specific magnetisation for the compound with bilateral elastic embedding (sketch iii)) is represented by the curve (f) in Fig. 7. The elastic modulus of phase 2 is the same as for curve c) of the unilateral embedding. The lateral extension induced by the $\mathrm{H}$-field is restricted due to phase 3 resulting in a reduction of the magnetisation. The curve (f) is thus below the curve (c). Sketch (iv) in Fig. 6 shows the FE model of the compound, where the colour pattern represents the magnetisation which, of course, only exists in the $\mathrm{CoFe}_{2} \mathrm{O}_{4}$ phase.
Figure 8 (left) shows the specific magnetisation and induction, respectively, vs. the magnetic field. In these calculations, case (b) in Fig. 5 is considered, where the boundaries are free of stress. The constitutive model being related to the local coordinate system, which is equal to the global coordinate system in this case, $\mathrm{B}$ and $\mathrm{m}$ are aligned with the H-field. The induction exhibits a linear increase for sufficiently large $\mathrm{H}$-fields, while the magnetisation approaches saturation. This essential feature is due to the introduction of the material related quantities $\mu_{11}^{0}$ or $\varpi$ into the model according to Eq. (14). The magnetic induction $B$ is once more shown in Fig. 8 (right), skipping the negative axes. The graph also shows the magnetic permeabilities based on stress or strain as independent variables according to the constitutive Eqs. (7) and (18). Obviously, there is no significant deviation between $\tilde{\mu}$ and $\bar{\mu}$. The magnification of the two plots in Fig. 8 (right) illustrates the magnitudes in detail. The consistency of the constitutive model is underlined, the magnetic induction not being influenced at all by the variance of the two magnetic permeabilities. Due to the traction-free mechanical boundary conditions, the green dotted line for $\tilde{\mu}$ represents the slope of the solid line $B(H)$.

Figure 9 shows the results of magnetostriction, i.e., both axial (left) and transverse (right) strain plotted vs. the magnetic field. Additionally, the magnetostrictive coefficients $\tilde{q}_{1 j}$ and $\bar{q}_{1 j}$ related to strain and stress as independent variables, respectively, are presented. The boundary conditions are the same as in Fig. 8. It is obvious from the dashed blue line that the coefficient $\bar{q}_{11}$ is always negative. The coefficient $\tilde{q}_{11}$, on the other hand, takes both signs, depending on the magnitude of the H-field. For sufficiently large magnetic fields, the axial coefficients asymptotically tend to zero, thus representing
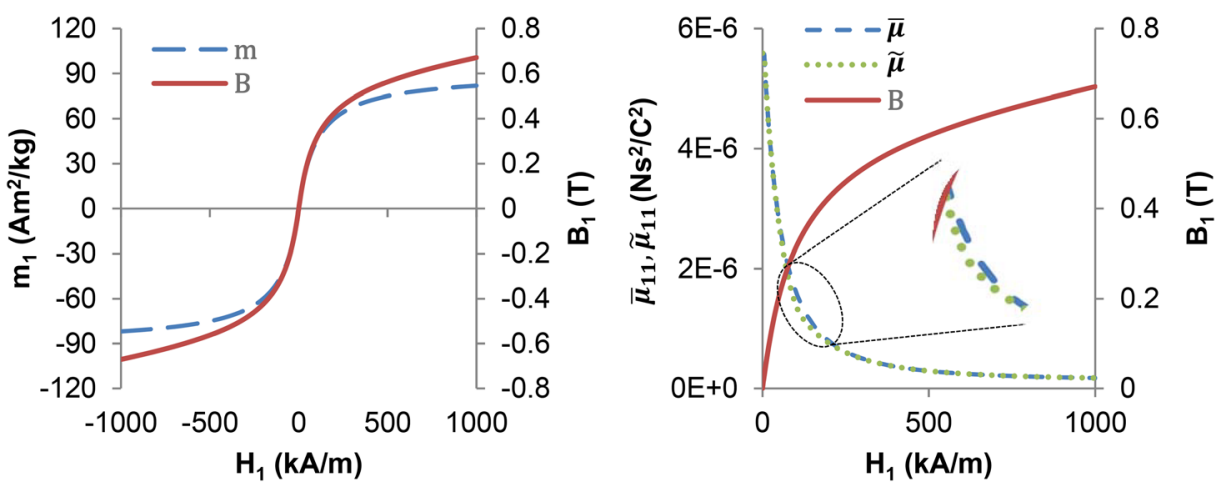

FIG. 8. Numerical results for stressfree boundaries, left: magnetic induction $B_{1}$ and specific magnetisation $m_{1}$, right: magnetic induction and magnetic permeabilities $\tilde{\mu}_{11}$ and $\bar{\mu}_{11}$ vs. the magnetic field $H_{1}$. 


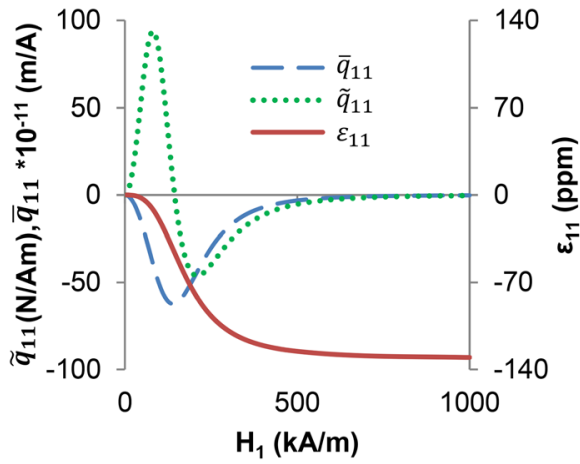

the saturation of strain. Again, both definitions of the magnetostrictive coefficient $\tilde{q}_{1 j}$ and $\bar{q}_{1 j}$, together with the respective associated constitutive equations, yield identical values of strain. Due to the stress-free boundaries, the dashed lines $\bar{q}_{1 j}$ provide the derivatives of the strain vs. magnetic field curves. The $\bar{q}_{12}$ thus has to vanish for large H-fields, while $\tilde{q}_{12}$ intersects the abscissa.

\section{CONCLUSIONS}

A phenomenological constitutive model for soft ferromagnetic materials are presented, exhibiting nonlinear reversible behaviour. Due to intended applications with respect to multiferroic composites, dielectric properties are included in the constitutive approach. The material model, allowing for multiaxial multifield loading has been implemented within a finite element context to be able to investigate the complex boundary value problems. Verifications of the modelling approach under various loading and boundary conditions, including those of compound structures with integrated non-functional elastic phases, demonstrate its capability of appropriately and efficiently describing the reversible ferromagnetic material behaviour.

\section{APPENDIX: MATERIAL DATA AND THEIR IDENTIFICATION}

The coefficients of cobalt ferrite, as listed in Table I, are found in (Li and Dunn, 1998) and (Tang and Yu, 2009).

Additionally, the quantities in Table II have been identified for the nonlinear constitutive model.

Due to the fact, that an appropriate value of $\mu_{11}^{r}$ could not be found in literature, $\mu_{11}^{r}=5$ has been chosen based on similar ferromagnetic materials.

Table III illustrates the procedure of how to identify the parameters of the constitutive model based on experimental and numerical curves.

TABLE I. Material properties of $\mathrm{CoFe}_{2} \mathrm{O}_{4}$.

\begin{tabular}{lc}
\hline \hline & $\mathrm{CoFe}_{2} \mathrm{O}_{4}$ \\
\hline$c_{11}(\mathrm{GPa})$ & 269.5 \\
$c_{12}(\mathrm{GPa})$ & 170 \\
$c_{22}(\mathrm{GPa})$ & 286 \\
$c_{23}(\mathrm{GPa})$ & 173 \\
$c_{44}(\mathrm{GPa})$ & 45.3 \\
$\kappa_{11}(\mathrm{C} / \mathrm{Vm})$ & $0.093 \times 10^{-9}$ \\
$\kappa_{22}(\mathrm{C} / \mathrm{Vm})$ & $0.080 \times 10^{-9}$ \\
\hline \hline
\end{tabular}

TABLE II. Parameters of the material model adapted to the constitutive behaviour of $\mathrm{CoFe}_{2} \mathrm{O}_{4}$.

\begin{tabular}{lccc}
\hline \hline No. & Parameter & Unit & $\mathrm{CoFe}_{2} \mathrm{O}_{4}$ \\
\hline 1 & $\eta_{1}$ & $\ldots$ & $-131 \times 10^{-6}$ \\
2 & $\eta_{2}$ & $\ldots$ & $106 \times 10^{-6}$ \\
3 & $\zeta_{1}^{0}$ & $\mathrm{~A}^{3} / \mathrm{m}^{3}$ & $5.5 \times 10^{15}$ \\
4 & $\zeta_{2}^{0}$ & $\mathrm{~A}^{3} / \mathrm{m}^{3}$ & $2.1 \times 10^{15}$ \\
5 & $\zeta_{1}^{\sigma}$ & $\mathrm{A}^{3} / \mathrm{Nm}$ & $9 \times 10^{10}$ \\
6 & $\rho$ & $\mathrm{T}$ & 0.6 \\
7 & $\xi^{0}$ & $\mathrm{~N} / \mathrm{Vs}$ & $1 \times 10^{5}$ \\
8 & $\xi^{\sigma}$ & $\mathrm{m}^{2} / \mathrm{Vs}$ & 0.3 \\
9 & $\hat{\xi}_{0}^{\sigma}$ & $\mathrm{m}^{2} / \mathrm{Vs}$ & 6 \\
10 & $\varpi$ & $\cdots$ & 50 \\
\hline \hline
\end{tabular}

TABLE III. Identification of the constitutive model parameters.

\begin{tabular}{lcc}
\hline \hline No. & Parameter & Calibration \\
\hline 1 & $\eta_{1}$ & Saturation strain $\varepsilon_{11}^{s}$ at $\sigma_{i j}=0$ \\
2 & $\eta_{2}$ & Saturation strain $\varepsilon_{22}^{s}$ at $\sigma_{i j}=0$ \\
3 & $\zeta_{1}^{0}$ & $\varepsilon_{11}-H-\operatorname{curve}$ at $\sigma_{i j}=0$ \\
4 & $\zeta_{2}^{0}$ & $\varepsilon_{11}-H-\operatorname{curve}$ at $\sigma_{i j}=0$ \\
5 & $\zeta_{1}^{\sigma}$ & curve at $\sigma_{i j}=$ const $\neq 0$ \\
6 & $\rho$ & Saturation magnetisation $\left(\right.$ converted in to T) at $\sigma_{i j}=0$ \\
7 & $\xi^{0}$ & $m-H-\operatorname{curve}$ at $\sigma_{i j}=0$ \\
8 & $\xi^{\sigma}$ & $m-H-$ curve at $\sigma_{i j}=$ const $\neq 0$ \\
9 & $\hat{\xi}_{0}^{\sigma}$ & Sigidly clamped $\left.: d \sigma_{i j} \neq 0\right)$ \\
10 & $\varpi$ & Slope of $\vec{B}$ at saturation of magnetisation $\left(* \mu_{0}^{-1}\right)$ at $\sigma_{i j}=0$ \\
\hline \hline
\end{tabular}

For a plane stress state, the values of $\sigma_{11}^{s}$ and $\sigma_{22}^{s}$ are calculated as follows:

$$
\begin{aligned}
& \sigma_{11}^{s}=c_{11} \eta_{1}+c_{12} \eta_{2}-\frac{c_{12}}{c_{22}}\left(c_{12} \eta_{1}+c_{23} \eta_{2}\right), \\
& \sigma_{22}^{s}=c_{12} \eta_{1}+c_{22} \eta_{2}-\frac{c_{23}}{c_{22}}\left(c_{12} \eta_{1}+c_{23} \eta_{2}\right) .
\end{aligned}
$$

Avakian, A., Gellmann, R., and Ricoeur, A., "Nonlinear modeling and finite element simulation of magnetoelectric coupling and residual stress in multiferroic composites," Acta Mech. 226(8), 2789-2806 (2015).

Avakian, A. and Ricoeur, A., "Constitutive modeling of nonlinear reversible and irreversible ferromagnetic behaviors and application to multiferroic composites," J. Intell. Mater. Syst. Struct. 27(18), 2536-2554 (2016).

Bergmann, L. and Schaefer, C., Lehrbuch der Experimentalphysik (de Gruyter, Berlin/New York, 2005), Vol. 6.

Bhame, S. D. and Joy, P. A., "Tuning of the magnetostrictive properties of $\mathrm{CoFe}_{2} \mathrm{O}_{4}$ by $\mathrm{Mn}$ substitution for Co," J. Appl. Phys. 100(11), 113911 (2006). 
Bhame, S. D. and Joy, P. A., "Magnetic and magnetostrictive properties of manganese substituted cobalt ferrite," J. Phys. D: Appl. Phys. 40(11), 3263-3267 (2007).

Bhame, S. D. and Joy, P. A., "Effect of sintering conditions and microstructure on the magnetostrictive properties of cobalt ferrite," J. Am. Ceram. Soc. 91(6), 1976-1980 (2008).

Bibes, M. and Barthélémy, A., "Multiferroics: Towards a magnetoelectric memory," Nat. Mater. 7(6), 425-426 (2008).

Bozorth, R. M., Ferromagnetism (Van Nostrand, New York, 1951).

Buchanan, G. R., "Layered versus multiphase magneto-electro-elastic composites," Composites, Part B 35(5), 413-420 (2004).

Carman, G. P. and Mitrovic, M., "Nonlinear constitutive relations for magnetostrictive materials with applications to 1-D problems," J. Intell. Mater. Syst. Struct. 6(5), 673-683 (1995).

Du Trémolet de Lacheisserie, E., Gignoux, D., and Schlenker, M., Magnetism. Materials and Applications (Springer, New York, 2005).

Eerenstein, W., Wiora, M., Prieto, J., Scott, J. F., and Mathur, N., "Giant sharp and persistent converse magnetoelectric effects in multiferroic epitaxial heterostructures," Nat. Mater. 6(5), 348-351 (2007).

Etier, M. F., Shvartsman, V. V., Stromberg, F., Landers, J., Wende, H., and Lupascu, D. C., "Synthesis and magnetic properties of cobalt ferrite nanoparticles," In 2011 MRS Fall Meeting \& Exhibit (Symposium Q: Magnetoelectric Composites), Boston, USA, 28 November-2 December 2011 (2012), p. 1398.

Feltin, N. and Pileni, M. P., "New technique for synthesizing iron ferrite magnetic nanosized particles," Langmuir 13(15), 3927-3933 (1997).

Fiebig, M., "Revival of the magnetoelectric effect," J. Phys. D: Appl. Phys. 38(8), R123-R152 (2005).

Jackson, J. D., Classical Electrodynamics (John Wiley \& Sons, New York, 1998).

Hill, N., "Why are there so few magnetic ferroelectrics?" Journal of Physical Chemistry B 104(29), 6694-6709 (2000).

Kallenbach, E., Eick, R., Quendt, P., Ströhla, T., Feindt, K., Kallenbach, M., and Radler, O., Elektromagnete. Grundlagen, Berechnung, Entwurf und Anwendung (Vieweg+Teubner, Wiesbaden, 2012).

Kiefer, B. and Lagoudas, D. C., "Phenomenological modeling of ferromagnetic shape memory alloys,” Proc. SPIE 5387, 164-176 (2004).

Kittel, C., Einführung in die Festkörperphysik (Oldenbourg, München, 2006), Vol. 14.

Li, J. Y. and Dunn, M. L., "Micromechanics of magnetoelectroelastic composite materials: Average fields and effective behavior," J. Intell. Mater. Syst. Struct. 9(6), 404-416 (1998).

Linnemann, K., Klinkel, S., and Wagner, W., "A constitutive model for magnetostrictive and piezoelectric materials," Int. J. Solids Struct. 46, 1149-1166 (2009).

Lu, A. H., Salabas, E. L., and Schüth, F., "Magnetic nanoparticles: synthesis, protection, functionalization, and application," Angew. Chem. 46(8), 1222-1244 (2007).
Lu, X. Y., Li, H., and Wang, B., "Theoretical analysis of electric, magnetic and magnetoelectric properties of nano-structured multiferroic composites,” J. Mech. Phys. Solids 59(10), 1966-1977 (2011).

Miehe, C., Kiefer, B., and Rosato, D., "An incremental variational formulation of dissipative magnetostriction at the macroscopic continuum level," Int. J. Solids Struct. 48(13), 1846-1866 (2011a).

Miehe, C., Rosato, D., and Kiefer, B., "Variational principles in dissipative electro-magneto-mechanics: A framework for the macro-modeling of functional materials," Int. J. Numer. Methods Eng. 86(10), 1225-1276 (2011b).

Mohaideen, K. K. and Joy, P. A., "Studies on the effect of sintering conditions on the magnetostriction characteristics of cobalt ferrite derived from nanocrystalline powders," J. Eur. Ceram. Soc. 34(3), 677-686 (2014).

Morrish, A. H., The Physical Principles of Magnetism (IEEE Press, New York, 2001)

Nan, C. W., "Magnetoelectric effect in composites of piezoelectric and piezomagnetic phases," Phys. Rev. B 50(9), 6082-6088 (1994).

Nan, C. W., Bichurin, M. I., Dong, S. X., Viehland, D., and Srinivasan, G., "Multiferroic magnetoelectric composites: Historical perspective, status, and future directions," J. Appl. Phys. 103(3), 031101 (2008).

Scott, J. F., "Data storage: Multiferroic memories," Nat. Mater. 6(4), 256-257 (2007).

Shi, P., Jin, K., and Zheng, X., "A general nonlinear magnetomechanical model for ferromagnetic materials under a constant weak magnetic field," J. Appl. Phys. 119, 145103 (2016).

Stefanita, C. G., Magnetism. Basics and Applications (Springer, Heidelberg/ New York, 2012).

Tang, T. and Yu, W., "Micromechanical modeling of the multiphysical behavior of smart materials using the variational asymptotic method," Smart Mater. Struct. 18(12), 125026 (2009).

Vanderlinde, J., Classical Electromagnetic Theory (Springer, Dordrecht, Netherlands, 2005).

Wan, Y., Fang, D., and Hwang, K. C., "Nonlinear constitutive relations for magnetostrictive materials," Int. J. Nonlinear Mech. 38(7), 1053-1065 (2003)

Wan, Y. P. and Zhong, Z., "Vibration analysis of Tb-Dy-Fe magnetostriction actuator and transducer," Int. J. Mech. Mater. Des. 1, 95-107 (2004).

Wang, D., Wang, L., and Melnik, R., “A differential algebraic approach for the modeling of polycrystalline ferromagnetic hysteresis with minor loops and frequency dependence," J. Magn. Magn. Mater. 410, 144-149 (2016).

Xu, H., Pei, Y., Fang, D., and Ai, S., "An energy-based dynamic loss hysteresis model for giant magnetostrictive materials," Int. J. Solids Struct. 50(5), 672-679 (2013).

Zheng, X. J. and Liu, X. E., “A nonlinear constitutive model for Terfenol-D rods,” J. Appl. Phys. 97, 053901 (2005). 